Gil Flores, J., de Besa Gutiérrez, M. y Garzón Umerenkova, A. (2020). ¿Por qué procrastina el alumnado universitario? Análisis de motivos y caracterización del alumnado con diferentes tipos de motivaciones. Revista de Investigación Educativa, 38(1), 183-200.

DOI: http://dx.doi.org/10.6018/rie.344781

\title{
¿Por qué procrastina el alumnado universitario? Análisis de motivos y caracterización del alumnado con diferentes tipos de motivaciones
}

\section{Why do university students procrastinate? An analysis of the reasons and characterization of students with different reasons for procrastination}

\author{
Javier Gil Flores*, Manuel Rafael De Besa Gutiérrez** y Angélica Garzón Umerenkova*** \\ *Departamento MIDE. Universidad de Sevilla (España) \\ **Facultad de Ciencias de la Educación. Universidad de Cádiz (España) \\ ****Fundación Universitaria Konrad Lorenz. Bogotá (Colombia)
}

\begin{abstract}
Resumen
La procrastinación académica es una conducta que afecta a un elevado porcentaje del alumnado universitario, por lo que ha sido profusamente tratada en la literatura científica. Aunque se ha estudiado ampliamente la frecuencia de procrastinación y su relación con otras variables, son más escasos los trabajos centrados en los motivos que llevan a procrastinar. En el presente estudio analizamos las razones para procrastinar en una muestra de 433 estudiantes de la Universidad de Cádiz, utilizando para ello la Procrastination Assessment Scale-Students (PASS). Las respuestas obtenidas han permitido llevar a cabo una clasificación del alumnado según sus motivaciones. Los resultados indican que pueden diferenciarse tres tipos de alumnos, los cuales procrastinarían respectivamente por una inadecuada respuesta a las exigencias de la tarea, por temor e inseguridad, o por búsqueda de excitación y dependencia de otros. Cada uno de estos tipos ha sido caracterizado en función de rasgos personales y académicos. Edad, año cursado, planificación del tiempo, intensidad de procrastinación, autoconcepto como estudiante
\end{abstract}

Correspondencia: Javier Gil Flores. jflores@us.es, Facultad de Ciencias de la Educación, Departamento MIDE, C/ Pirotecnia, s/n, 41013 Sevilla. 
y autoeficacia son factores relevantes en la diferenciación entre tipos de alumnado con distintos motivos para procrastinar. En cambio, las variables sexo, simultanear los estudios con un trabajo remunerado y rendimiento académico no presentan efectos significativos. A partir de los resultados obtenidos, se hace una valoración de los mismos y se reflexiona sobre la intervención dirigida a reducir la procrastinación académica en estudiantes universitarios.

Palabras clave: procrastinación académica; motivos para procrastinar; educación superior; tipos de alumnado.

\section{Abstract}

Academic procrastination is a behaviour that affects a high percentage of university students, which has been extensively addressed in the scientific literature. Although the frequency of procrastination and its relation to other variables has been studied extensively, there are fewer studies focused on the motives that lead to procrastination. In the present study, we analyse the reasons for procrastinating in a sample of 433 students from the University of Cádiz, using the Procrastination Assessment Scale-Students (PASS). The answers obtained have allowed to carry out a classification of the students according to their motivations. The results indicate that three types of students can be differentiated, which would procrastinate respectively for an inadequate response to the demands of the task, due to fear and insecurity, or for a search for excitement and dependence on others. Each of these types has been characterized according to personal and academic characteristics. Age, academic year, time planning, intensity of procrastination, self-concept as a student and self-efficacy are relevant factors in the differentiation between types of students with different motives to procrastinate. On the other hand, the variables gender, simultaneous studies with paid work and academic performance do not have significant effects. We make a discussion of the results obtained and we reflect on the intervention aimed at reducing academic procrastination in university students.

Keywords: academic procrastination; reasons for procrastination; Higher Education; types of students.

\section{Introducción}

Desde el punto de vista psicológico, la procrastinación es definida como un fallo autorregulatorio en el individuo (Pychyl \& Flett, 2012; Rozental \& Carlbring, 2014; Steel, 2007), del que se derivan efectos no deseados para los estudiantes, su bienestar y su rendimiento. Procrastinar tareas académicas supone diferir o demorar su realización hasta incluso rebasar los límites temporales fijados, aun sabiendo que habría sido mejor cubrir dichas tareas en el momento fijado para ello. Sin embargo, el aplazamiento es un componente necesario, pero no suficiente para definir la procrastinación. Este retraso se considera procrastinación cuando va unido a malestar o consecuencias negativas para los individuos que lo practican (Martinčeková \& Enright, 2018; Steel \& Klingsieck, 2015). Los procrastinadores pueden experimentar frustración, descontento con el retraso o sentimiento de culpa (Corking, Yu \& Lindt, 2011; Hen \& Goroshit, 2018; Krause \& Freund, 2014). Se han encontrado evidencias de que la procrastinación frecuente se relaciona con problemas de ansiedad, estrés, salud física y mental (Kroese \& de Ridder, 2016; Sirois \& Tosti, 2012; Stead, Shanahan \& Neufeld, 2010). 
La prevalencia de procrastinación académica entre el alumnado universitario es elevada, mostrándose superior a la que se constata en la población general. Mientras que la procrastinación afecta a un $20 \%$ de la población adulta, en el ámbito universitario alcanza al 50\% (Rozental \& Carlbring, 2014), y ese mismo porcentaje señalan Day, Mensink y O'Sullivan (2000) cuando tratan de cuantificar al alumnado que procrastina de manera reiterada y problemática. Por encima de esas cifras, Steel y Ferrari (2013) indicaban que la demora de tareas académicas afecta a entre el 80\% y 95\% de los estudiantes universitarios, la mayor parte de los cuales reconocen ser procrastinadores.

La literatura ha estudiado el fenómeno de la procrastinación académica, analizando la relación entre esta conducta y una serie de variables, relativas tanto a características demográficas como a rasgos personales (Steel \& Klingsieck, 2016). El meta-análisis de Steel (2007) refleja que las mujeres procrastinan menos que los hombres, probablemente porque presentan mejores habilidades de manejo del tiempo (Durán-Aponte \& Pujol, 2013; Kaya, Kaya, Ozturk \& Kucuk, 2012) y tienden a esforzarse más que los hombres en sus actividades académicas (Clariana, 2013). Los resultados obtenidos en diversos trabajos apuntan a una mayor procrastinación entre los más jóvenes (Rozental \& Carlbring, 2014; Steel, 2007), y que la mayor tendencia a procrastinar se da en la transición del bachillerado a los estudios universitarios (Clariana, Gotzens, Badia \& Cladellas, 2012). Se ha descrito también la relación entre procrastinación y rendimiento académico, ampliamente analizada en el meta-análisis de Kim y Seo (2015). Los resultados de este estudio revelan una relación negativa entre ambas variables, de tal manera que el rendimiento académico obtenido por los estudiantes que habitualmente procrastinan es inferior y sus trabajos tienden a presentar un nivel más bajo de calidad.

En el presente trabajo, nuestro interés se dirige hacia las causas de la procrastinación. El estudiante podría demorar las tareas de manera activa, con el propósito de poner a punto las habilidades requeridas y lograr un mejor rendimiento o, en otro sentido, para evitar las consecuencias desagradables que podrían derivarse de las mismas, como por ejemplo la obtención de una mala calificación. De acuerdo con ello, trabajos anteriores han destacado la relación de la procrastinación con un exceso de perfeccionismo que lleva a revisar una y otra vez una tarea sin darla por acabada, debido al miedo a cometer errores (Pychyl \& Flett, 2012), con el deseo de trabajar bajo presión y sentirse activado a realizar la tarea cuando el tiempo se acaba (Chun \& Choi, 2010) o con la ansiedad que suscita la evaluación, y particularmente el miedo al fracaso (Zarick \& Stonebraker, 2009). La falta de una motivación intrínseca, derivada del interés por aprender o el disfrute en la realización de una tarea, constituye un factor que favorece la procrastinación (Steel, 2007). Otra variable asociada es la autoeficacia; cuando los estudiantes tienen una baja confianza en sus habilidades académicas y en sus expectativas de éxito son más vulnerables a incurrir en procrastinación (Wäschle, Allgaier, Lachner, Fink \& Nückles, 2014).

Además de las causas intrínsecas, las razones para la procrastinación pueden ser externas al individuo, hallándose en factores contextuales. Uno de estos factores tiene que ver con el profesorado y su desempeño docente. Así, la procrastinación se incrementaría cuando el profesor reduce el nivel de exigencia, está dispuesto a negociar los plazos de entrega de las tareas y es más flexible a la hora de calificar (Schraw, Wadkins \& Olafson, 2007), o cuando no proporciona instrucciones claras sobre en qué consisten 
y cómo han de llevarse a cabo las tareas que propone a sus estudiantes (Ackerman \& Gross, 2005). Igualmente, cuando los estudiantes perciben las tareas como difíciles y que requieren un elevado esfuerzo, tienden a presentar mayores tasas de conductas procrastinadoras (Ferrari, Mason \& Hammer, 2006). Sin embargo, tareas excesivamente fáciles pueden resultar aburridas y poco interesantes, promoviendo la procrastinación, por lo que las tareas planteadas habrían de lograr un equilibrio entre su nivel de exigencia y la posibilidad de que el estudiante pueda realizarlas (Steel, 2007).

Otro importante factor contextual de la procrastinación es la influencia de los pares. Klingsieck, Grund, Schmid y Fries (2013) constataron la influencia de otras personas, particularmente familiares o amigos, cuando éstas exhiben conductas procrastinadoras. En el trabajo de Foster y Frikters (2010), los estudiantes reconocen que su nivel de esfuerzo en una tarea se ve influenciado por los esfuerzos que perciben en sus compañeros, con el consiguiente impacto sobre las conductas procrastinadoras.

A pesar de la amplitud de trabajos de investigación que se han venido realizando en las últimas décadas acerca de la procrastinación académica, todavía puede afirmarse que hay una falta de conocimiento acerca de las razones por las que los estudiantes procrastinan (Visser, Korthagen \& Schonenboom (2018). También es un hecho que la investigación sobre los motivos para procrastinar se ve limitada por el instrumento empleado para evaluar la procrastinación general y académica, siendo pocos los que contienen un abanico amplio de motivos subyacentes a este tipo de conductas. Además, las técnicas de análisis empleadas no favorecen la categorización o discriminación de los distintos tipos de procrastinadores. Como mencionan Grunschel, Patrzek y Fries (2013), los análisis normalmente realizados en el área están centrados en las variables, analizando las relaciones entre ellas en lugar de clasificar a los individuos en grupos homogéneos según sus motivaciones. En consecuencia, la investigación sobre la procrastinación académica ha puesto el énfasis sobre la relación entre las características de los individuos y la frecuencia o intensidad de sus conductas procrastinadoras, dejando al margen el análisis de tipologías de alumnado procrastinador. Saliendo al paso de esta situación, el presente estudio se ha centrado en la caracterización de los tipos de estudiantes procrastinadores atendiendo a los motivos que les llevan a procrastinar.

\section{Método}

Metodológicamente, el trabajo adopta un diseño no experimental o ex post facto, a través de métodos de encuesta, con carácter transversal y basado en la comparación de grupos.

\section{Objetivos}

En este trabajo nos hemos planteado como objetivo analizar los motivos por los que el alumnado universitario procrastina sus tareas académicas, como punto de partida para establecer una tipología de alumnado en función de sus razones para procrastinar. Además, pretendemos caracterizar a los diferentes tipos de procrastinadores partiendo de rasgos personales y académicos comúnmente considerados en la investigación sobre la procrastinación académica. 


\section{Población y Muestra}

Teniendo como marco la población estudiantil universitaria, la muestra empleada la constituyen 433 estudiantes de la Universidad de Cádiz, seleccionados a través de muestreo no probabilístico y atendiendo a criterios de accesibilidad. Un $79.0 \%$ de la muestra son mujeres, siendo el $21.0 \%$ restante hombres. Las edades oscilaban entre los 18 y 51 años, con una media de 21.57 años (D.T: 3,99). Los participantes realizaban en el curso 2017-18 estudios de grado en Educación Infantil (17.6\%), Educación Primaria (44.9\%) y Psicología (37.5\%).

\section{Variables e Instrumentos}

Los datos sobre motivos para procrastinar se han obtenido mediante la escala Procrastination Assessment Scale-Students (PASS), desarrollada por Solomon y Rothblum (1984). Consta de 44 ítems divididos en dos secciones. Para medir los motivos utilizamos la versión adaptada al español por Garzón y Gil (2017), y concretamente la segunda sección del instrumento, formada por 26 ítems sobre las razones cognitivo-conductuales que llevan al estudiante a procrastinar. Al diseñar la PASS, Solomon y Rothblum (1984) plantearon una situación de procrastinación, consistente en el retraso al entregar un trabajo final de curso, y propusieron trece posibles razones para explicar esta conducta en los estudiantes, haciendo corresponder a cada una de ellas dos ítems. Las respuestas se expresan mediante una escala de cinco puntos, desde no refleja mis motivos en absoluto (1) hasta los refleja perfectamente (5). La puntuación en los trece motivos para procrastinar se tomó como suma de las obtenidas en los dos ítems correspondientes. Tras realizar un análisis factorial exploratorio (AFE), Solomon y Rothblum (1984) solo consideraron dos de los siete factores obtenidos: uno que agrupó las razones de ansiedad, perfeccionismo y baja autoconfianza, y otro que agrupó las razones de aversión a la tarea y pereza. El resto de las razones aparecieron agrupadas en factores con autovalores menores o iguales a 1.50 y fueron descartados por las autoras. En su trabajo, no aportaron valores de la fiabilidad del instrumento; con los datos del presente estudio se ha obtenido un alfa de Cronbach de 0.86 .

Con la intención de retener la totalidad de los motivos expresados en la PASS, hemos utilizado los datos obtenidos en el presente estudio para llevar a cabo un AFE mediante componentes principales, con rotación varimax. Previamente, se comprobó que la matriz de correlaciones era adecuada, con $\mathrm{KMO}=.807$ y $\mathrm{p}<.001$ en el test de esfericidad de Barlett. Los motivos quedaron agrupados en tres factores que explican un $54.29 \%$ de la varianza total (ver saturaciones en Tabla 1). Las mayores saturaciones en el primer factor corresponden a los motivos ansiedad, perfeccionismo y poca autoconfianza, que fueron agrupados por Solomon y Rothblum (1984) en el factor miedo al fracaso. A estos se unen la dificultad para tomar decisiones, para expresarlas o mantenerlas y el miedo al éxito, motivos todos ellos que indican inseguridad. Hemos denominado a este primer factor "temor e inseguridad". El segundo factor incluye los motivos relacionados con el rechazo a la tarea y la pereza, que constituían el segundo de los factores considerados por Solomon y Rothblum (1984), añadiéndose la inadecuada gestión del tiempo y la no aceptación del control por parte de otros. Este factor representa motivos referidos a una 
“inadecuada respuesta a las exigencias de la tarea". Por último, en el tercer factor están presentes la excitación que suscita el riesgo de que se cumpla el tiempo límite para la tarea, la influencia de los pares y la necesidad de obtener ayuda de otros. Se trata de un factor motivacional relativo a "búsqueda de excitación y dependencia de otros".

Tabla 1

Saturaciones de los motivos para procrastinar en los tres factores motivacionales tras la rotación varimax

\begin{tabular}{|c|c|c|c|}
\hline Motivos para procrastinar & Factor 1 & Factor 2 & Factor 3 \\
\hline Ansiedad ante la evaluación & .795 & & \\
\hline Perfeccionismo & .763 & & \\
\hline Poca autoconfianza & .607 & .371 & \\
\hline Baja asertividad & .598 & & \\
\hline Dificultad para tomar decisiones & .593 & & \\
\hline Miedo al éxito & .584 & & .384 \\
\hline $\begin{array}{l}\text { Tendencia a sentirse desbordado y pobre gestión del } \\
\text { tiempo }\end{array}$ & & .800 & \\
\hline Rebelión contra el control & .352 & .727 & \\
\hline Aversión a la tarea & & .686 & \\
\hline Pereza & & .642 & .431 \\
\hline Asunción de riesgos & & & .777 \\
\hline Influencia de pares & & .432 & .566 \\
\hline Dependencia y búsqueda de ayuda & .326 & & .537 \\
\hline
\end{tabular}

Nota: Se han suprimido las saturaciones inferiores a.300

Junto a los motivos para procrastinar, se ha considerado un conjunto de variables personales y académicas, utilizadas para caracterizar al alumnado que difiere en sus motivos. Una de estas variables es la intensidad de procrastinación académica. Siguiendo la propuesta de Rothblum, Solomon y Murakami (1986) se midió a partir de dos ítems incluidos en la primera parte de la PASS que aluden a la demora en estudiar para exámenes hasta el último momento, recogiendo respectivamente con qué frecuencia se produce esta conducta y con qué frecuencia ello supone un problema. Ambos se responden con una escala de cinco puntos, que van desde nunca (1) hasta siempre (5), y la intensidad de procrastinación se obtiene como suma de los dos ítems, generando una escala de 2 a 10 puntos.

Otra variable utilizada es la autoeficacia académica, entendida como la percepción que tiene el individuo sobre su capacidad para llegar a actuar de manera exitosa en el ámbito académico. Se midió utilizando la Escala de Autoeficacia Percibida Específica de Situaciones Académicas (EAPESA), desarrollada por Palenzuela (1983). Originalmente constaba de 10 ítems con una escala de respuesta de cuatro puntos (nunca, algunas 
veces, bastantes veces y siempre), aunque su autor aconsejó la eliminación del ítem 9, lo que mejoraba las propiedades psicométricas del instrumento. Estudios posteriores (Domínguez et al., 2012; García et al., 2010) corroboran esta recomendación, confirman el carácter unidimensional y obtienen índices de fiabilidad entre 0.87 y 0.92 . Con los datos del presente estudio, la fiabilidad medida por alfa de Cronbach asciende a 0.91. Las puntuaciones en autoeficacia académica se han obtenido como promedio de los ítems que componen la escala.

El resto de las variables utilizadas han sido medidas a través de un cuestionario diseñado al efecto. Además de los rasgos demográficos sexo y edad, el cuestionario recogía información sobre las siguientes variables:

Experiencia universitaria. Diferencia entre estudiantes de nuevo ingreso, que cursan el primer año de estudios, y estudiantes de cursos superiores.

- Dedicación a los estudios. Indica si los estudiantes desempeñan o no un trabajo remunerado simultáneamente a sus estudios.

- Planificación del trabajo académico. Recoge si los encuestados planifican su tiempo ofreciendo una escala de respuesta con cinco modalidades, desde nunca (1) hasta siempre (5).

- Rendimiento académico. A partir del autoinforme de los estudiantes sobre sus calificaciones en las materias cursadas durante el primer cuatrimestre del curso 2017-18, el rendimiento se obtuvo como promedio de dichas calificaciones (medido en una escala de 1 a 4).

- Autoconcepto como estudiante. La percepción sobre sí mismo se valoró al preguntar al alumnado cómo de buenos o malos estudiantes se consideran, ofreciendo una escala numérica desde 0 (muy malo) hasta 10 (muy bueno).

A excepción de los motivos para procrastinar, cuyo análisis será objeto del primer bloque de resultados, la tabla 2 recoge las variables implicadas en el estudio. Se presentan los estadísticos media y desviación típica, o bien porcentajes, dependiendo del nivel de medida.

Tabla 2

Estadísticos descriptivos para las variables utilizadas en la caracterización del alumnado

\begin{tabular}{llc}
\multicolumn{1}{c}{ Variables } & \multicolumn{1}{c}{ Categorías } & Descriptivos \\
\hline \multirow{2}{*}{ Sexo } & 1=Mujer & $79.0 \%$ \\
Edad & 2=Hombre & $21.0 \%$ \\
Experiencia universitaria & 1=Primer curso & M=21.57; DT=3.99 \\
& 2=Cursos superiores & $39.1 \%$ \\
Dedicación al estudio & 1=Trabajo simultáneo & $60.9 \%$ \\
& 2=Sin trabajo simultáneo & $27.8 \%$ \\
\hline
\end{tabular}




\begin{tabular}{ll}
\hline Planificación del tiempo (valores de 1 a 5) & $\mathrm{M}=3.55 ; \mathrm{DT}=0.99$ \\
Intensidad de procrastinación (valores de 2 a 10) & $\mathrm{M}=6.78 ; \mathrm{DT}=1.70$ \\
Rendimiento académico (valores de 1 a 4 ) & $\mathrm{M}=1.67 ; \mathrm{DT}=0.65$ \\
Autoconcepto como estudiante (valores de 0 a 10) & $\mathrm{M}=7.11 ; \mathrm{DT}=1.24$ \\
Autoeficacia académica (valores de 1 a 4 ) & $\mathrm{M}=2.86 ; \mathrm{DT}=0.57$ \\
\hline
\end{tabular}

Nota: $M=$ Media $; D T=$ Desviación típica

\section{Procedimiento de recogida y análisis de datos}

Para la recogida de datos se solicitó colaboración a profesorado que impartía docencia en titulaciones de la Facultad de Ciencias de la Educación, de la Universidad de Cádiz. Al hacerlo, se procuró que en la muestra aceptante estuvieran representados estudiantes de diferentes titulaciones, tanto de primer curso como de cursos posteriores. La aplicación de los instrumentos tuvo lugar en marzo de 2018, dentro de las aulas, empleando aproximadamente quince minutos. Los estudiantes participaron de manera voluntaria, y se les garantizó anonimato y confidencialidad de sus respuestas.

El análisis de datos se ha iniciado con una descripción de los motivos para procrastinar, basada en medias y desviaciones típicas para los trece motivos contemplados. Utilizando las puntuaciones factoriales en los tres factores motivacionales, calculadas mediante el método de regresión, se ha realizado un análisis de conglomerados mediante el que se clasifica al alumnado, generando una tipología según sus motivaciones. Conforme a la naturaleza cuantitativa de las variables, se tomó la distancia euclídea al cuadrado como medida de similitud entre individuos, y se utilizó el método de Ward como procedimiento de agregación. Finalmente se han caracterizado los tipos de alumnado resultantes, recurriendo a diferentes técnicas. En el caso de variables medidas a nivel de intervalo, se aplica el análisis de la varianza, comparando las medias alcanzadas en los grupos de estudiantes identificados. Previamente se comprobó la homoscedasticidad de varianzas a través de la prueba de Levene, manteniéndose la hipótesis nula de igualdad de varianzas para estas variables cuando se fija un nivel de significación $\alpha=.05$, con la única excepción de la variable edad, para la cual la hipótesis se mantiene con $\alpha=.01$. Para las variables a nivel nominal, el contraste se ha basado en el estadístico chi-cuadrado. En todos los análisis se empleó el programa SPSS 24.

\section{Resultados}

\section{Motivos del alumnado para procrastinar}

De acuerdo con los resultados mostrados en la Tabla 3, entre las mayores puntuaciones medias alcanzadas por los motivos para demorar la entrega de un trabajo final se encuentran la pereza (5.85), junto a sentirse desbordado por la tarea y manejar pobremente el tiempo (6.86). Las razones más importantes de la procrastinación habría que buscarlas, por tanto, en una falta de esfuerzo por parte del estudiante y en una mala gestión del tiempo. Ambos constituyen aspectos vinculados a una inadecuada forma 
de responder a las tareas académicas. En este factor motivacional se incluyen también dos motivos que se encuentran entre los más destacados: la aversión a la tarea (5.79) y la rebelión ante el control (5.45). Estos implican una reacción de rechazo a realizar un trabajo que requiere esfuerzo, o a tener que hacerlo por indicación de otra persona y dentro de un plazo fijado.

Tabla 3

Estadísticos descriptivos correspondientes a los motivos del alumnado para procrastinar

\section{Motivos para procrastinar Media Desviación típica}

Factor temor e inseguridad

$\begin{array}{lll}\text { Ansiedad ante la evaluación } & 6.10 & 2.37 \\ \text { Perfeccionismo } & 5.61 & 2.24 \\ \text { Poca autoconfianza } & 5.11 & 2.13 \\ \text { Baja asertividad } & 4.33 & 1.89 \\ \text { Dificultad para tomar decisiones } & 5.68 & 1.54 \\ \text { Miedo al éxito } & 2.99 & 1.42\end{array}$

Factor inadecuada respuesta a las exigencias de la tarea

Tendencia a sentirse desbordado y pobre gestión del tiempo

$6.86 \quad 1.84$

Rebelión contra el control

Aversión a la tarea

5.79

Pereza

Factor búsqueda de excitación y dependencia de otros

Asunción de riesgos

Influencia de pares

Dependencia y búsqueda de ayuda

5.31

1.83

Nota: La escala de puntuaciones en cada motivo comprende valores de 2 a 10

Algo menos de peso tienen los motivos que se enmarcan en el factor temor e inseguridad, destacando la ansiedad ante la evaluación (6.10), la dificultad para tomar decisiones (5.68) y el perfeccionismo (5.61). Por tanto, la preocupación por las calificaciones que puedan obtenerse, la indecisión a la hora de concretar el trabajo y el temor de no ver satisfechas unas elevadas expectativas que el propio estudiante se habría fijado son motivos que en cierta medida llevan a procrastinar.

Finalmente, las razones menos relevantes al justificar la procrastinación tienen que ver con la búsqueda de excitación y la dependencia de otros. En este bloque se incluyen dos de los tres motivos que menos reflejan la posición de los estudiantes. Concretamente, 
la asunción de riesgos (2.93) y la influencia de pares (4.12). De acuerdo con ello, el deseo de sentir la presión de que el tiempo se acaba y asumir el reto de realizar la tarea en el último momento es algo que no mueve al alumnado a procrastinar. Y tampoco es frecuente que las tareas se demoren por esperar a que otros compañeros también las realicen o por anteponer otras actividades cediendo a la presión de los amigos.

\section{Tipología de estudiantes en función de sus motivos para procrastinar}

La obtención de una tipología de estudiantes se ha basado en el análisis de conglomerados. A partir de las puntuaciones factoriales en los tres factores bajo los que se agrupan los motivos para procrastinar (temor e inseguridad, inadecuada respuesta a las exigencias de la tarea, y búsqueda de excitación y dependencia de otros), se han calculado las distancias entre individuos, tomando como medida la distancia euclídea al cuadrado. La aplicación del método de Ward como procedimiento de agregación ha permitido obtener una clasificación jerárquica de los participantes. Tras el examen de esta, se ha optado por realizar una partición de la muestra en cuatro clases, logrando así un equilibrio óptimo entre la separación de las clases y la homogeneidad dentro de las mismas. Cada clase se corresponde con un tipo de alumnado con distintas motivaciones para procrastinar. Los cuatro tipos resultantes aparecen recogidos en la Tabla 4, donde se incluye su descripción a partir de las medias logradas en cada uno de los factores motivacionales. Al tratarse de puntuaciones factoriales, y por tanto de variables centradas y reducidas, el valor 0 puede tomarse como punto de corte para discriminar entre puntuaciones negativas, que representan menor puntuación en el factor, y puntuaciones positivas, que representan mayor puntuación.

Tabla 4

Medias para los factores motivacionales en cada tipo de estudiante

\begin{tabular}{lcccc}
\hline \multicolumn{1}{c}{ Factores motivacionales } & $\begin{array}{c}\text { Tipo 1 } \\
(\mathbf{n = 9 7 )}\end{array}$ & $\begin{array}{c}\text { Tipo 2 } \\
(\mathbf{n = 5 6 )}\end{array}$ & $\begin{array}{c}\text { Tipo 3 } \\
(\mathbf{n = 1 4 4 )}\end{array}$ & $\begin{array}{c}\text { Tipo 4 } \\
(\mathbf{n = 1 0 8 )}\end{array}$ \\
\hline Miedo e inseguridad & 0.62 & -0.15 & 0.36 & -0.96 \\
Inadecuada respuesta a las exigencias de la tarea & -0.92 & -0.08 & 0.83 & -0.24 \\
Búsqueda de excitación y dependencia de otros & -0.16 & 1.70 & -0.20 & -0.47 \\
\hline
\end{tabular}

Nota: Las puntuaciones factoriales se calcularon por el método de regresión.

El tipo 1 corresponde a alumnado para el que temor e inseguridad (Media=0.62) constituyen el principal motivo que explica su conducta procrastinadora, mientras que los otros dos grupos de motivaciones tienen menor peso. Los estudiantes incluidos en el tipo 2 alcanzan una puntuación media de 1.70 en el segundo factor y puntuaciones negativas en los restantes. Este alumnado destaca claramente por procrastinar en busca de la excitación que genera el riesgo de que se cumpla el tiempo límite para la tarea, y por depender de otras personas que le presten ayuda o simplemente sirvan como 
referencia para sus conductas. Como ya se apuntó en el apartado anterior al describir los motivos para procrastinar, estas razones tienen poca relevancia entre el alumnado, lo que explica que en este tipo hayan quedado incluido únicamente 56 estudiantes. El tipo 3 se identifica con procrastinar por no ser capaz de dar una respuesta adecuada a las tareas que han de afrontarse (Media=0.83), debido a una falta de esfuerzo, una deficitaria gestión del tiempo o el rechazo a la propia tarea, a realizarla de manera impuesta y dentro de un plazo límite. Finalmente, el tipo 4 incluye estudiantes con puntuaciones negativas, y que por tanto se identifican poco con las razones para procrastinar, sin que puedan ser asociados a ninguna de estas razones en particular. De cara a posteriores análisis, retendremos la tipología de estudiantes que representan los tipos 1 a 3, los cuales quedan asociados a cada uno de los tres factores motivacionales.

\section{Rasgos asociados a los diferentes tipos de alumnado procrastinador}

La caracterización de los tres tipos de alumnado procrastinador, identificados a partir de sus razones para procrastinar, se ha basado en la comparación de los valores alcanzados en cada uno de esos tipos por una serie de variables personales y académicas. En la tabla 5 se presentan los resultados de estas comparaciones, basadas en el análisis de la varianza y la prueba de chi-cuadrado.

Tabla 5

Contraste de rasgos personales y académicas entre los tres tipos de alumnado

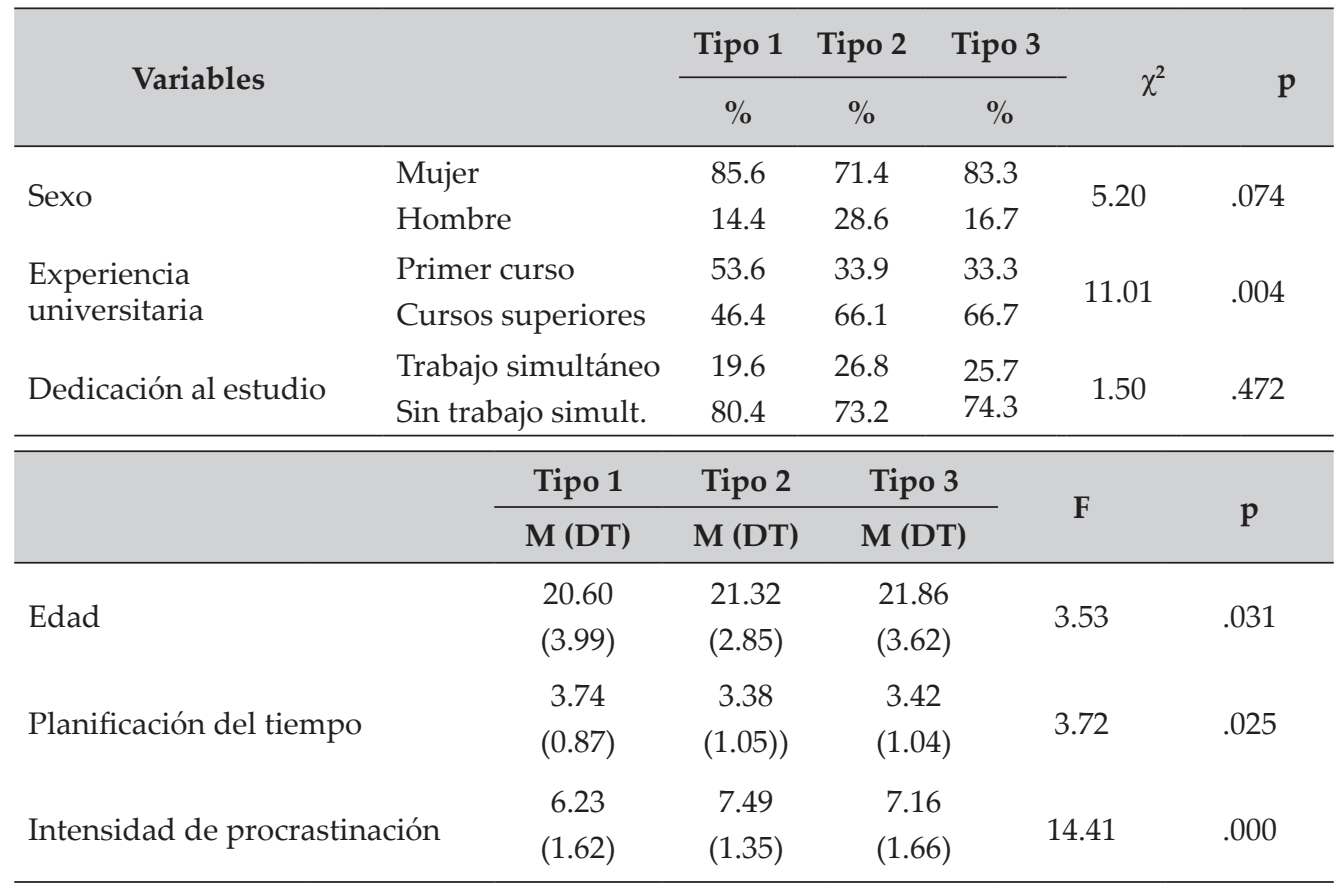




\begin{tabular}{lcccccc}
\hline & Tipo 1 & Tipo 2 & Tipo 3 & \multirow{2}{*}{ F } & p \\
\cline { 2 - 4 } & $\mathbf{M}$ (DT) & M (DT) & M (DT) & & \\
\hline \multirow{2}{*}{ Rendimiento académico } & 1.60 & 1.59 & 1.66 & & \\
& $(0.66)$ & $(0.64)$ & $(0.61)$ & & .645 \\
Autoconcepto como estudiante & 7.32 & 7.09 & 6.76 & & \\
& $(1.09)$ & $(1.10)$ & $(1.36)$ & & \\
Autoeficacia académica & 2.85 & 3.01 & 2.68 & & .002 \\
& $(0.60)$ & $(0.53)$ & $(0.55)$ & 7.31 & .001 \\
\hline
\end{tabular}

Los rasgos que más diferencian a los tres tipos de alumnado son la intensidad con que procrastinan $(\mathrm{p}=.000)$, la autoeficacia académica $(\mathrm{p}=.001)$, el autoconcepto como estudiante (.002) y la experiencia universitaria $(\mathrm{p}=.004)$. En menor medida resultan significativas $(\mathrm{p}<.05)$ las diferencias en función de la planificación de tiempo y la edad. En función de estos resultados, entre los procrastinadores por temor e inseguridad (tipo 1) se encuentra el alumnado con menos experiencia. Concretamente, entre ellos hay más alumnado de nuevo ingreso (53.6\% en este grupo frente al $33.9 \%$ y $33.3 \%$ en los dos restantes grupos), consecuentemente más joven (media=20.60 años), aunque planifica su tiempo de trabajo (media $=3.74$ ), presenta menor intensidad de procrastinación (media $=6.23$ ) y posee un elevado autoconcepto como estudiante (media=7.32). El perfil de quienes procrastinan por responder inadecuadamente a las exigencias de las tareas planteadas (tipo 2) se concreta en un estudiante que presenta mayor intensidad de procrastinación (media $=7.49)$ y que planifica menos el tiempo (media=3.38), aunque con más experiencia en la universidad (el 66.1\% en segundo curso o posteriores) y, probablemente por ello, con una mayor confianza en su capacidad para realizar las tareas académicas (media de 3.01 en autoeficacia). Finalmente, los estudiantes que demoran su trabajo porque buscan la excitación y dependen de otros para afrontar sus tareas (tipo 3) presentan como rasgos característicos los niveles más bajos de autoconcepto y autoeficacia académica (medias de 6.76 y 2.68 respectivamente). Se trata de estudiantes con mayor edad (media=21.86 años) y experiencia universitaria $(66.7 \%$ en cursos superiores a primero de carrera).

No han resultado significativas $(\alpha=.05)$ las diferencias entre los tres tipos de estudiantes en lo que respecta a sexo, rendimiento académico y realización de algún trabajo remunerado al tiempo que estudian.

\section{Discusión y conclusiones}

El primer objetivo de este trabajo es describir los motivos por los que procrastina el alumnado universitario. Los resultados obtenidos sitúan en primera línea la falta de una adecuada gestión del tiempo, la ansiedad ante la evaluación y la pereza. No en vano la procrastinación puede ser entendida como un fallo en la autorregulación del individuo (Pychyl \& Flett, 2012; Rozental \& Carlbring, 2014), asociada por tanto 
a dificultades para gestionar el tiempo. En lo que respecta a la ansiedad ante la evaluación, es decir el temor del estudiante a que se evalúe su nivel de competencia o desempeño y a las consecuencias negativas que puedan derivarse de ello, se trata de un tópico ampliamente tratado en la literatura, y de manera especial cuando se concreta en la ansiedad ante pruebas y exámenes (Álvarez, Aguilar \& Lorenzo, 2012; Constantin, English \& Mazmanian, 2018). En el caso de entrega de trabajos finales, a los que se asignará una calificación que puede ser decisiva para la superación o no de una asignatura, no resulta extraño que la ansiedad ante la evaluación derive en conductas de evitación de la tarea y de demora, constituyendo uno de los principales motivos para procrastinar. Junto a la mala gestión del tiempo y a la ansiedad, entre los motivos más frecuentes, se encuentra la falta de disposición del alumnado para esforzarse ante tareas académicas tales como la realización de un trabajo final de curso.

Tratando de salir al paso de estas razones para la demora, la intervención dirigida a reducir las conductas procrastinadoras habría de incluir planes de desarrollo de las habilidades de gestión del tiempo como mecanismo preventivo más eficaz (Chen, 2011; Häfner, Oberst \& Stock, 2014). Existen técnicas, tanto presenciales como virtuales, individuales o grupales, que se vienen mostrando exitosas para la disminución de la procrastinación académica. Por ejemplo, se han usado técnicas para el aumento de la flexibilidad mental Glick y Orsillo (2015); e intervenciones cognitivo-conductuales que fortalecen la autorregulación, tales como el establecimiento de rutinas y calendarios, la exposición a los eventos que generan ansiedad y llevan al estudiante a procrastinar o el manejo de las creencias irracionales, entre otras (Rozental et al., 2014). Este tipo de intervenciones ha logrado efectos positivos en cuanto a reducción de la procrastinación, que permanecen estables en el tiempo (Van Eerden \& Kingsieck, 2018). En contextos académicos, el entrenamiento en habilidades para la gestión del tiempo no solo conlleva la disminución de la procrastinación y la mejora del rendimiento, sino que también produce una reducción en la ansiedad de los estudiantes, mejora la salud mental y física, mejora el manejo de las relaciones sociales y recreativas, y en general, conduce a una mejora de la calidad de vida de los estudiantes (Kaya et al., 2012).

Por otra parte, la sobrecarga de tareas exigidas simultáneamente al alumnado universitario limita el tiempo disponible para su realización, lo que supone un problema añadido a la mala gestión temporal. En este sentido, las instituciones universitarias han de insistir en mecanismos de coordinación que permitan distribuir en el tiempo las tareas requeridas al alumnado en las diferentes asignaturas que conforman un curso académico, evitando la concentración de trabajos en un período corto y racionalizando los plazos de entrega. A la mejora en la gestión del tiempo se añadiría la necesidad de aminorar la ansiedad ante la evaluación, para lo que se han propuesto terapias psicológicas e intervenciones educativas dirigidas a los estudiantes, basadas en el entrenamiento en las habilidades necesarias para preparar y afrontar las tareas implicadas (Huntley, Young, Jha \& Fisher, 2016). En lo que respecta a la organización de los procesos de enseñanza y aprendizaje por parte del profesorado, la reducción de la ansiedad podría verse favorecida por la clarificación de los sistemas de evaluación, de tal manera que los estudiantes conozcan con detalle cuáles son los criterios de evaluación y calificación. El tercero de los motivos, la pereza, debe sus- 
citar una reflexión sobre los valores de esfuerzo y trabajo, que han de desarrollarse entre el alumnado desde las etapas educativas más tempranas, y sobre la necesidad de hacer atractivas, interesantes y útiles las tareas propuestas, convirtiéndolas en tareas auténticas, conectadas con futuros desempeños profesionales y académicos, con las que se consiga captar el interés de los estudiantes y lograr su motivación e implicación en las mismas.

En relación con el segundo de los objetivos planteados, se ha conseguido diferenciar al menos tres tipos de estudiantes en función de sus razones para procrastinar. Estos se identifican con quienes responden inadecuadamente a las exigencias que plantean las tareas académicas, quienes sienten temor o inseguridad, y quienes buscan la excitación o se ven influidos por compañeros y amigos. Un cuarto grupo, con importante representación entre el alumnado, no se identifica de manera clara con las razones para procrastinar aquí consideradas. Los rasgos personales y académicos asociados a cada tipo han llevado a señalar perfiles de estudiantes adscritos a estas motivaciones. La intervención orientadora dirigida a evitar problemas de procrastinación puede preventivamente dirigirse a sujetos que se ajustan a los perfiles señalados, con especial atención a quienes procrastinan por inadecuada respuesta a las exigencias de la tarea, que constituyen el colectivo más numeroso. Además, la identificación del tipo de estudiantes procrastinadores permitiría una intervención diferenciada, adaptada para dar respuesta a los motivos que en cada caso llevan a procrastinar.

Al caracterizar a los tipos de alumnado según sus motivaciones, el rendimiento académico no ha resultado ser un rasgo diferenciador. El meta-análisis llevado a cabo por Kim y Seo (2015) concluyó que existe una relación negativa entre procrastinación y rendimiento. A partir de nuestros resultados, podemos añadir que la relación entre rendimiento y procrastinación no habría de verse alterada por los motivos que llevan a procrastinar. Del mismo modo, aunque la literatura ha señalado que el alumnado que simultanea sus estudios con el desempeño de un trabajo remunerado podría ser menos procrastinador (Forbus, Newbold \& Mehta, 2010), la simultaneidad de los estudios con un trabajo no supone un factor diferenciador entre quienes señalan distintos motivos para procrastinar.

El trabajo que aquí hemos presentado puede suponer una aportación relevante en el estudio de la procrastinación académica, al abordar el análisis de las razones para procrastinar, tema sobre el que recientemente se ha señalado un déficit de conocimiento (Visser, Korthagen \& Schonenboom, 2018). Además, nos hemos centrado en los individuos y en su clasificación en grupos homogéneos en función de sus motivaciones, como reclamaban Grunschel. Patrzek y Fries. (2013). Como limitaciones podemos señalar el carácter no probabilístico de la muestra, que impide la generalización de resultados a la totalidad de alumnado universitario, y las limitaciones propias de los procedimientos de autoinforme, sujetos al sesgo derivado de la subjetividad o de una posible falta de sinceridad por parte de quienes responden. Líneas futuras de investigación podrían dirigirse a valorar la consistencia de los perfiles de estudiantes aquí caracterizados cuando se trabaja con muestras de otros contextos geográficos o de otras áreas de enseñanza universitaria. Una vía interesante sería la exploración de otros motivos para procrastinar, dado que en el presente estudio se ha identificado un grupo de estudiantes que no se ve reflejado en las motivaciones propuestas. 
Además, dada la importancia de una mala gestión del tiempo como principal motivo para procrastinar, la implementación y evaluación de programas orientados a mejorar la gestión del tiempo, con el fin de comprobar sus efectos en términos de reducción de las conductas procrastinadoras en el alumnado universitario, se configura como otro interesante campo de trabajo.

\section{Referencias}

Ackerman, D.S. y Gross, B.L. (2005). My instructor made me do it: task characteristics of procrastination. Journal of Marketing Education. 27(1), 5-13. doi:10.1177/0273475304273842

Álvarez, J., Aguilar, J.M. y Lorenzo, J.J. (2012). La ansiedad ante los exámenes en estudiantes universitarios: relaciones con variables personales y académicas. Electronic Journal of Research in Educational Psychology, 10(1), 333-354.

Chen, P. (2011). Guiding College students to develop academic self-regulatory skills. Journal of College Teaching and Learning, 8(9), 29-33. doi:10.19030/tlc.v8i9.5642

Chun, A.H. y Choi, J.N. (2010) Rethinking procrastination: positive effects of "active" procrastination behavior on attitudes and performance. The Journal of Social Psychology, 145(3), 245-264, doi:10.3200/SOCP.145.3.245-264

Clariana, M. (2013). Personalidad, Procrastinación y Conducta Deshonesta en Alumnado de distintos Grados Universitarios. Electronic Journal of Research in Educational Psychology, 11(2), 451-472. doi:10.14204/ejrep.30.13030

Clariana, M., Gotzens, C., Badia, M. y Cladellas, R. (2012). Procrastinación y engaño académico desde la Secundaria hasta la Universidad. Electronic Journal of Research in Educational Psychology, 10(2), 737-754.

Constantin, K., English, M.M. y Mazmanian, D. (2018). Anxiety, depression, and procrastination among students: rumination plays a larger mediating role than worry. Journal of Rational-Emotive \& Cognitive-Behavior Therapy, 36(1), 15-27. doi:10.1007/s10942017-0271-5

Corkin, D.M., Yu, S.L. y Lindt, S.F. (2011). Comparing active delay and procrastination from a self-regulated learning perspective. Learning and Individual Differences, 21(5), 602-606. doi:10.1016/j.lindif.2011.07.005

Day, V., Mensink, D. y O'Sullivan, M. (2000). Patterns of academic procrastination. Journal of College Reading and Learning, 30(2), 120-134. doi:10.1080/10790195.2000 .10850090

Domínguez, S., Villegas, G. Yauri, C., Mattos. E. y Ramírez F. (2012). Propiedades psicométricas de una escala de autoeficacia para situaciones académicas en estudiantes universitarios peruanos. Revista de Psicología- Universidad Católica San Pablo. 2(1), 27-39.

Durán-Aponte, E. y Pujol, L. (2013). Manejo del tiempo académico en jóvenes que inician estudios en la Universidad Simón Bolívar. Revista Latinoamericana de Ciencias Sociales, Niñez y Juventud, 11(1), 93-108. doi:10.11600/1692715x.1115080812

Ferrari, J., Mason, C. y Hammer, C. (2006). Procrastination as a predictor of task perceptions: Examining delayed and non-delayed tasks across varied deadlines. Individual Differences Research, 4(1), 28-36. 
Forbus, P., Newbold, J. y Mehta, S. (2010). A study of non-traditional and traditional students in terms of their time management behaviors, stress factors, and coping strategies. Proceedings of the Academy of Educational Leadership, 15(2), 67-71.

Foster, G. y Fritjers, P. (2010). Students' beliefs about peer effects. Economic Letters, 108(3), 260-263. doi:10.1016/j.econlet.2010.05.020

García, J.M., Inglés. C.J., Torregrosa, M.S., Ruiz, C., Díaz, Á, Pérez, E. y Martínez, M.C. (2010). Propiedades psicométricas de la Escala de Autoeficacia Percibida Específica de Situaciones Académicas en una muestra de estudiantes españoles de Educación Secundaria Obligatoria. European Journal of Education and Psychology, 3(1), 61-74. doi:10.30552/ejep.v3i1.51

Garzón, A. y Gil, J. (2017). Propiedades psicométricas de la versión en español de la prueba Procrastination Assessment Scale-Students (PASS). Revista Iberoamericana de Diagnóstico y Evaluación Psicológica, 43(1), 149-163. doi:10.21865/RIDEP43_149

Glick, D. y Orsillo, S. (2015). An investigation of the efficacy of acceptance-based behavioral therapy for academic procrastination. Journal of Experimental Psychology: General, 144(2), 400-409. doi:10.1037/xge0000050

Grunschel, C., Patrzek, J. y Fries, S. (2013). Exploring the Reasons and Consequences of Academic Procrastination: An Interview Study. European Journal of Psychology of Education, 28(3), 841-861. doi:10.1007/s10212-012-0143-4

Häfner, A., Oberst, V. y Stock, A. (2014). Avoiding procrastination through time management: an experimental intervention study. Educational Studies, 40(3), 352-360. doi $: 10.1080 / 03055698.2014 .899487$

Hen, M. y Goroshit, M. (2018). The effects of decisional and academic procrastination on students' feelings toward academic procrastination. Current Psychology, 1-8. doi:10.1007/s12144-017-9777-3

Huntley, C.D., Young, B., Jha, V. y Fisher, P.L. (2016). The efficacy of interventions for test anxiety in university students: A protocol for asystematic review and meta-analysis. International Journal of Educational Research, 77, 92-98. doi:10.1016/j. ijer.2016.03.001

Kaya, H., Kaya, N., Palloş, A. Ö. y Küçük, L. (2012). Assessing time-management skills in terms of age, gender, and anxiety levels: A study on nursing and midwifery students in Turkey. Nurse Education in Practice, 12(5), 284-288. doi:10.1016/j. nepr.2012.06.002

Kim, K.R. y Seo, E.H. (2015). The relationship between procrastination and academic performance: A meta-analysis. Personality and Individual Differences, 82, 26-33. doi:10.1016/j.paid.2015.02.038

Klingsieck, K.B., Grund, A., Schmid, S. y Fries, S. (2013). Why students procrastinate: A qualitative approach. Journal of College Student Development, 54(4), 397-412. doi:10.1353/csd.2013.0060

Krause, K. y Freund, A.M. (2014). Delay or procrastination - A comparison of self-report and behavioral measures of procrastination and their impact on affective well-being. Personality and Individual Differences, 63, 75-80. doi:10.1016/j.paid.2014.01.050

Kroese, F., and de Ridder, D. (2016). Health behaviour procrastination: a novel reasoned route towards self-regulatory failure. Health psychology review, 10(3), 313-325. doi: 10.1080/17437199.2015.1116019 
Martinčeková, L. y Enright, R. D. (2018). The effects of self-forgiveness and shameproneness on procrastination: exploring the mediating role of affect. Current Psychology, 1-10. doi:10.1007/s12144-018-9926-3

Palenzuela, D. (1983). Construcción y validación de una escala de autoeficacia percibida específica de situaciones académicas. Análisis y Modificacion de Conducta, 9(21), 185-219.

Pychyl, T.A. y Flett, G.L. (2012). Procrastination and self-regulatory failure: An introduction to the special issue. Journal of Rational-Emotive $\mathcal{E}$ Cognitive-Behavior Therapy, 30(4), 203-212. doi:10.1007/s10942-012-0149-5

Rothblum, E., Solomon, L. y Murakami, J. (1986). Affective, cognitive, and behavioral differences between high and low procrastinators. Journal of Counseling Psychology, 33(4), 387-394. doi:10.1037/0022-0167.33.4.387

Rozental, A. y Carlbring, P. (2014). Understanding and treating procrastination: A review of a common self-regulatory failure. Psychology, 5(13), 1488-1502. doi:10.4236/ psych.2014.513160

Rozental, A., Forsström, D., Nilsson, S., Rizzo, A. y Carlbring, P. (2014) Group versus Internet-based cognitive-behavioral therapy for procrastination: Study protocol for a randomized controlled trial. Internet Interventions, 1(2), 84-89. doi:10.1016/j. invent.2014.05.005

Schraw, G., Wadkins, T. y Olafson, L. (2007). Doing the things we do: a grounded theory of academic procrastination. Journal of Education Psychology, 99(1), 12-25. doi:10.1037/0022-0663.99.1.12

Sirois, F. y Tosti, N. (2012). Lost in the moment? An investigation of procrastination, mindfulness, and well-being. Journal of Rational-Emotive $\mathcal{E}$ Cognitive-Behavior Therapy, 30(4), 237-248. doi:10.1007/s10942-012-0151-y

Solomon, L. y Rothblum, E. (1984). Academic procrastination: Frequency and cognitivebehavioral correlates. Journal of Counseling Psychology, 31(4), 503-509. doi:10.1037/00220167.31.4.503

Stead, R., Shanahan, M. y Neufeld, R. (2010). "I'll go to therapy, eventually": Procrastination, stress and mental health. Personality and Individual Differences, 49(3), 175-180. doi:10.1016/j.paid.2010.03.028

Steel, P. (2007). The nature of procrastination: A meta-analytic and theoretical review of quintessential self-regulatory failure. Psychological Bulletin, 133(1), 65-94. doi:10.1037/0033-2909.133.1.65

Steel, P. y Ferrari, J. (2013). Sex, education and procrastination: An epidemiological study of procrastinators' characteristics from a global sample. European Journal of Personality, 27(1), 51-58. doi:10.1002/per.1851

Steel, P. y Klingsieck, K. (2015). Procrastination. In J. D. Wright (Ed.), The international encyclopedia of the social \& behavioral sciences, 2nd Edition (pp. 73-78). Oxford: Elsevier.

Steel, P. y Klingsieck, K. B. (2016). Academic procrastination: psychological antecedents revisited. Australian Psychologist, 51(1), 36-46. doi:10.1111/ap.12173

Van Eerden, W. y Kingsieck, K.B. (2018), Overcoming procrastination? A meta-analysis of intevention studies. Educational Research Review, 25, 73-85. doi:10.1016/j.edurev.2018.09.002 
Visser, L, Korthagen, F.A.J. y Schoonenboom, J. (2018) Differences in learning characteristics between students with high, average, and low levels of academic procrastination: students' views on factors influencing their learning. Frontiers in Psychology, 9(808). doi:10.3389/fpsyg.2018.00808

Wäschle, K., Allgaier, A., Lachner, A., Fink, S. y Nückles, M. (2014). Procrastination and self-efficacy: Tracing vicious and virtuous circles in self-regulated learning. Learning and instruction, 29, 103-114. doi:10.1016/j.learninstruc.2013.09.005

Zarick, L. y Stonebraker, R. (2009). I'll do it tomorrow the logic of procrastination. College Teaching. 57(4), 211-215. doi:10.1080/87567550903218687

Fecha de recepción: 5 de octubre de 2018.

Fecha de revisión: 16 de octubre de 2018.

Fecha de aceptación: 21 de febrero de 2019. 